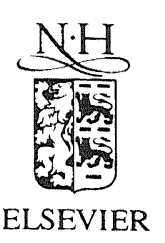

Applied Numerical Mathematics 19 (1995) 91-114

\title{
Explicit parallel block Cholesky algorithms on the CRAY APP
}

\author{
Margreet Nool * \\ CWI, P.O. Box 94079, 1090 GB Amsterdam, Netherlands
}

\begin{abstract}
In this paper we consider the CRAY APP, the Attached Parallel Processor of the CRAY S-MP, which consists of seven buses with each bus supporting up to 12 processing elements. Processing elements on different buses can communicate simultaneously with the shared main memory, but processing elements sharing the same bus can not, since only one processing element per bus can access memory at a given time. Applications with a high level of data reuse, or, with a high computation intensity, and applications being highly parallel are very suitable to run on the APP. An example of such an algorithm is matrix-matrix multiplication. We illustrate how the data traffic's restriction influences the performance and we discuss a performance model of the bus architecture, considering a change in processor speed, data traffic speed and cache contents.

Furthermore, two different algorithms for Cholesky factorization are discussed: a block left-looking algorithm and a block right-looking algorithm. The maximum achievable speed on the CRAY APP is mainly determined by the performance of the matrix-matrix multiplication. Parallelism is applied explicitly over the blocks, which makes it possible to concatenate different block operations in cache. The results obtained on CWI's APP (a machine having twenty-eight processing elements) indicate how block algorithms can be parallelized on machines with hundreds or thousands of processors.
\end{abstract}

Keywords: Software; Parallelization; Vectorization

\section{Introduction}

The Attached Parallel Processor APP of the CRAY S-MP is a system of 1,3,5 or 7 buses, each consisting of several processing elements. In the case of one processing element per bus, it is possible to obtain a speed-up which is close to the number of buses involved. However, in the case of two or more processing elements sharing the same bus, the speed-up is restricted by bus traffic, since only one processing element per bus can access main memory at a given time. For that reason, only parallel algorithms with a high computation intensity compared to data traffic are suitable to run efficiently on the APP. In earlier papers $[8,9,12]$, which report on APP performance, the speed-up was mainly limited by the number of buses rather than by the

\footnotetext{
${ }^{*}$ E-mail: greta@cwi.nl.
} 
number of processing elements. Here, we concentrate on such parallel applications, for which we actually obtain speed-ups higher than the number of buses. For that purpose we introduce the bus speed-up being a function of the execution time on B buses with one processor and that on $\mathrm{B}$ buses with $\mathrm{P}$ processors. Moreover, we consider a performance model of the APP, and we discuss the influence on the performance in case the speed of the processing elements or the speed of data transfer would increase. Finally, we consider the bus performance as a function of the cache contents. The CRAY APP is a rather exotic architecture, although its communication structure on one bus is similar to that in a network of workstations (which becomes an increasingly popular parallel computing environment). CRAY quotes several applications of the APP in image, sonar, and radar processing with speeds of 1-2.3 Gflop/s for one APP [13]. In [3] a speed of $548 \mathrm{Mflop} / \mathrm{s}$ is reported for the solution of a full linear system of order 1000 .

The matrix-matrix multiplication serves as a key in the first part of the paper dealing with the APP and its suitability for this operation. The Cholesky factorization, considered in the second part, is based on the matrix-matrix multiplication as well. Its performance is mainly determined by the speed of multiplication. The factorization can easily be parallelized, but during the progress of the factorization the level of parallelism decreases. We have found that it is difficult to predict how a given matrix should be divided into blocks to achieve optimal performance. Two Cholesky factorizations are compared: a left- and a right-looking algorithm. Though solving the same decomposition within the same number of steps, their performance differs significantly on a 28-processor CRAY APP.

The organization of the paper is as follows. In Section 2, we describe the CRAY APP configuration. In Section 3, we focus on the block matrix-matrix multiplication; it is performed in such a way that data traffic is minimal resulting in a high performance. A performance model of the CRAY APP is discussed in Section 4. In Section 5, two block Cholesky factorizations are described; much attention is paid to reducing the number of synchronization points leading to an increase of the performance. Finally, in Section 6, some conclusions are drawn.

\section{The CRAY APP configuration}

The CRAY APP, a multiple-bus, parallel processor, can be used in two modes: the client server programming model and the computer model. In the first model, the main program runs on the front end machine and highly parallel time-consuming parts of the program are run on the CRAY APP. In the latter model, which we consider in this paper, all operations are performed on the CRAY APP.

Its architecture, as illustrated in Fig. 1, consists of 4 to 84 processing elements and one to seven buses. The buses are connected to the main memory by a crossbar. This main memory is shared by all of the processing elements. An important restriction in bus traffic is that a given time only one processing element on each bus can access memory. As a consequence, for optimal use of the CRAY APP it is necessary to take care of a balanced bus traffic. An example of efficient bus usage is shown in Fig. 2. Due to the short load time compared to the computation time in cache, seven processing elements can be used efficiently. The addition of 


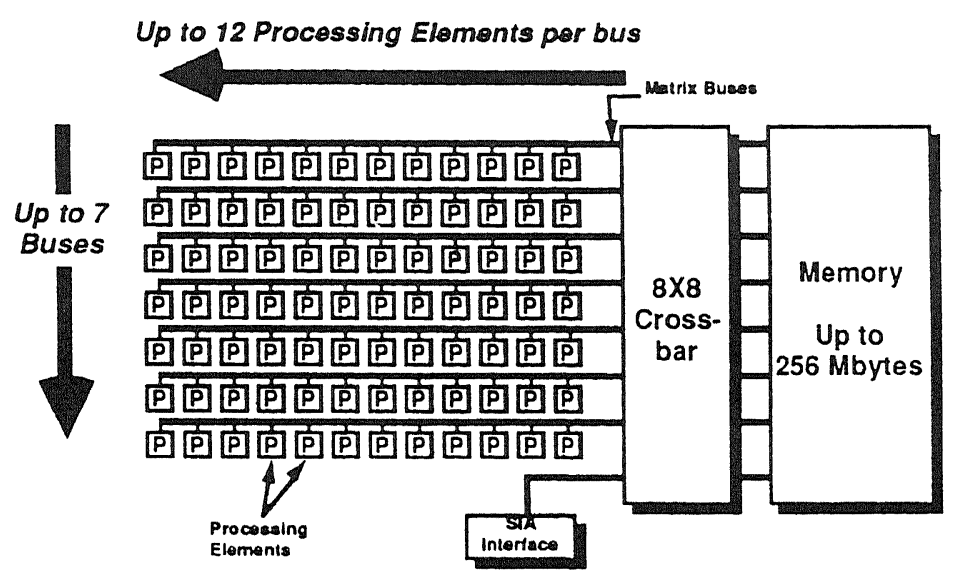

Fig. 1. CRAY APP architecture.

one or more processing elements to the bus will not result in a higher performance; several elements must wait to use the bus for loading. Obviously, the desired number of processing elements on a bus depends on the application.

There are two ways in which parallelism can be exploited on the CRAY APP:

- by using the auto-parallelizing feature of the compiler and insert directives for parallel processing wherever possible;

- by "bus-handoff" computing: hardware features are available for loading data into caches and for storing updated values into the main memory.

In this paper, we focus on the optimization of bus traffic and the reuse of data wherever possible. To this aim, we will investigate the application of "bus-handoff" computing.

\subsection{Some characteristics of the CRAY APP}

The CRAY APP at CWI, Amsterdam consists of seven buses, each with four processing elements. The data cache of each processing element is an 8-Kbyte memory chip so that for

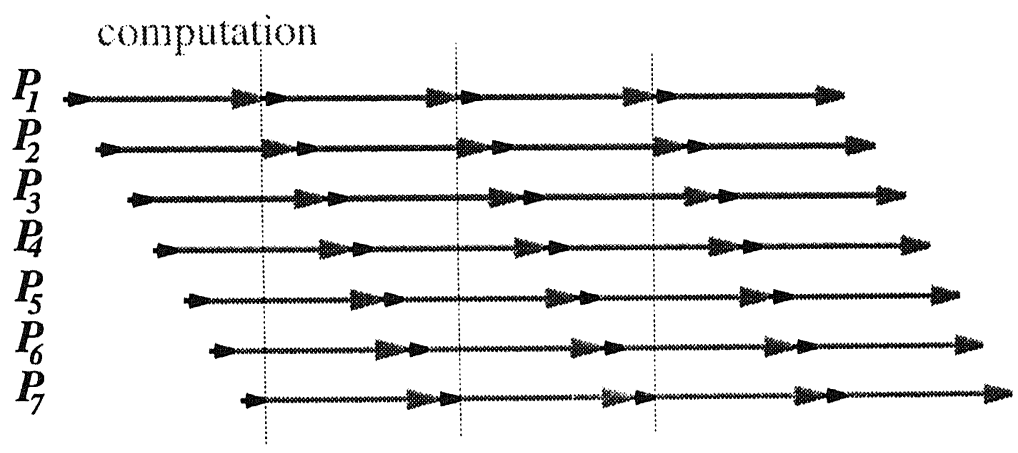

- data transfer

Fig. 2. Efficient bus usage of seven processors sharing the same bus. 
DOUBLE PRECISION arithmetic only 1 Kwords can be stored. This implies that for a matrix-matrix multiplication in cache involving two square operand matrices and one result matrix, the block size is restricted to 18 . The processing elements, based on the Intel i860 64-bit microprocessor, can reach a peak performance of $60 \mathrm{Mflop} / \mathrm{s}$ for DOUBLE PRECISION operations. However, when performing an equal number of DOUBLE PRECISION multiplies and adds, as in the matrix-matrix multiplication case, the maximum possible performance is 40 Mflop/s (not including data transfer). In addition, each bus provides a peak bandwidth of 160 Mbyte/s, i.e., 20 Mwords DOUBLE PRECISION words can be transferred per second. So, two flops and one data transfer can be done simultaneously.

\subsection{Some remarks on the software available on the CRAY APP}

From the previous section it seems easy to predict the performance of a parallel application, since everything needed for a good and reliable analysis is available, such as the data cache size, a performance peak of $40 \mathrm{Mflop} / \mathrm{s}$ as well as the data transfer time. Moreover, the CRAY APP has a very accurate clock, and timing results for a parallel application on the APP appear to be reproducible. On the CWI's configuration a peak Mflop rate of more than $1 \mathrm{Gflop} / \mathrm{s}$ should be possible. However, a reasonable performance can be obtained only by using cache programming and for this it is necessary to use the Extended Math Library routines [1]. Unfortunately, this library has only a small set of appropriate routines for numerical programming. It would be helpful if at least a set of Level 1 BLAS [10] routines were included in this library, but even commonly-used operations like inner products and daxpy operations are missing. In fact, only a limited set is available, and its usability is restricted by incomprehensible rules.

Consider, for instance, the routine _dvsvma, which performs the following operation on DOUBLE PRECISION data:

$$
\boldsymbol{D}(i)=A(i)+\boldsymbol{B} * \boldsymbol{C}(i), \quad \text { for } i=1, \ldots, n .
$$

The first restriction is that the vectors $\boldsymbol{A}$ and $\boldsymbol{D}$ may not share the same memory locations as they do in a daxpy operation. Therefore, this routine cannot be used as a simple daxpy operation without an extra vector copy and neither can any other Extended Math. Library routine. Secondly, the elements of $\boldsymbol{A}, \boldsymbol{C}$ and $\boldsymbol{D}$ must be consecutive elements in memory; an increment parameter is not allowed. The third restriction gives rise to most complications: $A, C$ and $D$ must be quad-word aligned. Operations can only be carried out in pipelined mode if the initial elements of the input data are stored on quad-word boundaries in cache. A word in the $w$ th position in cache is quad-word aligned, or on a quad-word boundary if $w \bmod 4=1$, as is described in [12]. For the DOUBLE PRECISION case either the odd or the even vector elements can be quad-word aligned; for the SINGLE PRECISION case only each fourth element of a vector satisfies this restriction.

Finally, we mention the DOUBLE PRECISION matrix-matrix multiplication routine _dmmm which has a speed of more than $25 \mathrm{Mflop} / \mathrm{s}$ on a single processor. This routine computes in cache

$$
\boldsymbol{C}=r * \boldsymbol{C}+s * \boldsymbol{A} * \boldsymbol{B},
$$


where $r$ and $s$ may be $-1,0$, or 1 . Besides the restriction that the memory occupied by $C$ should not intersect that occupied by $\boldsymbol{A}$ or $\boldsymbol{B}$, we must take into account that this routine is parameterized: all cache loads and stores are internal to the routine and all preloaded data in the cache will be lost. As a consequence, it does not seem to be possible to reuse the data, since each time the routine is called the cache will be refreshed. However, in Section 3.4 we show results of experiments where in certain cases data are still reused by this routine.

The absence of a good compiler for cache programming and the large number of restrictions makes programming very complicated and reduces the readability of programs considerably. Most executions do not achieve the performances claimed in the specifications. In Section 5.1.3, we return to this point.

\section{The matrix-matrix multiplication}

The matrix-matrix multiplication can be considered as one of the most suitable applications to run on the CRAY APP. Firstly, the multiplication is highly parallel; it can easily be divided into (many) parts which can run in parallel. Secondly, the (computation) intensity, defined by the ratio of the number of floating point operations to the number of words of data, is high. For a real matrix-matrix multiplication of the form $C=C+A * B$, the intensity is given by

$$
\text { Intensity }=\frac{\text { \#Flops }}{\text { \#Data Words Transferred }}=\frac{2 n^{3}}{4 n^{2}}=\frac{1}{2} n
$$

\subsection{Parallelism}

Let the real matrices $\boldsymbol{A}, \boldsymbol{B}$ and $\boldsymbol{C}$ of Fig. 3 have orders $m \times k, k \times n, m \times n$ and assume that $\mathrm{KB}, \mathrm{MB}$ and $\mathrm{NB}$ are proper divisors of $k, m$ and $n$, respectively, such that $\mathrm{K} \times \mathrm{KB}=k$, $\mathrm{M} \times \mathrm{MB}=m$ and $\mathrm{N} \times \mathrm{NB}=n$. Then, $A$ can be partitioned into a block matrix of $\mathrm{M} \times \mathrm{K}$ blocks of order $\mathrm{MB} \times \mathrm{KB}$. Matrices $\boldsymbol{B}$ and $\boldsymbol{C}$ can be partitioned analogously. An example of a block algorithm to perform the matrix-matrix multiplication $\boldsymbol{C}=\boldsymbol{C}+\boldsymbol{A} * \boldsymbol{B}$ is given by Fig. 4. The

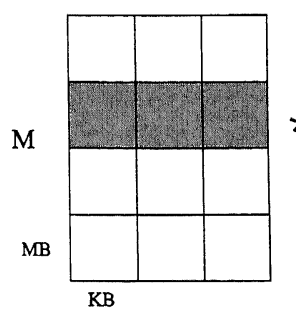

$\mathbf{A}(m, k)$
$\mathrm{N}$

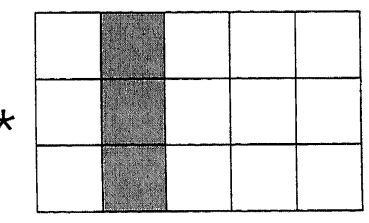

NB

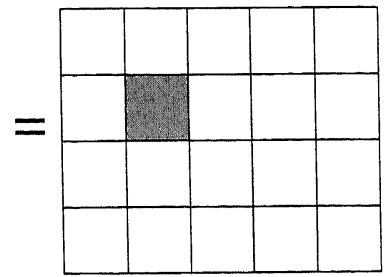

$\mathrm{C}(\boldsymbol{m}, \boldsymbol{n})$

Fig. 3. Matrix-matrix multiplication $A * B=C$; the matrices $A, B$ and $C$ are partitioned into blocks of order $\mathrm{MB} \times \mathrm{KB}, \mathrm{KB} \times \mathrm{NB}$ and $\mathrm{MB} \times \mathrm{NB}$, respectively. 


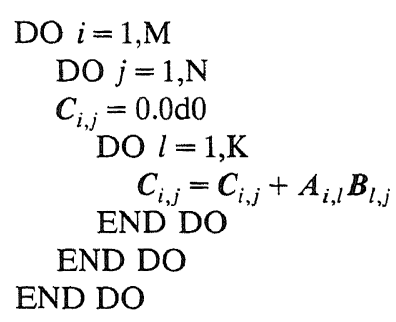

Fig. 4. Block matrix-matrix algorithm. Each block $\boldsymbol{A}_{i, l}, \boldsymbol{B}_{l, j}$ and $\boldsymbol{C}_{i, j}$ is of order $\mathrm{MB} \times \mathrm{KB}, \mathrm{KB} \times \mathrm{NB}$ and $\mathrm{MB} \times \mathrm{NB}$, respectively.

inner loop is not particularly suitable for parallel processing, since for each $l$ the same submatrix $C_{i, j}$ is updated. Introducing parallelism on the level of the $j$ loop results in updating the $\mathrm{N}$ block columns of $C$ in parallel. The introduction of parallelism on the outer loop level leads to concurrent computation of the block rows of $C$ and a level of parallelism equal to $\mathrm{M}$. Note, that if loops $i$ and $j$ are interchanged, parallelism over the block columns changes to parallelism over the block rows. However, there will be a significant difference in how operations are scheduled to the processing elements. The order of the operations will differ as well as the idle pattern if $\mathrm{M}$ or $\mathrm{N}$ are not a multiple of the number of processing elements. In [9], a block row and a block column scheme are considered and experiments on the CRAY APP display that the idle time becomes significant due to a lack of parallelism.

A higher level of parallelism and a possible reduction of idle time can be achieved by collapsing the middle and the outer loop. As a consequence, all computations on submatrices $C_{i, j}, i=1, \ldots, \mathrm{M}, j=1, \ldots, \mathrm{N}$, can be carried out independently, resulting in a level of Iarallelism of $\mathrm{M} \cdot \mathrm{N}$. Since each submatrix $C_{i, j}$ is stored once, the number of stores is minimal. In the other hand, the number of loads is high, since all submatrices of $\boldsymbol{A}$ and $\boldsymbol{B}$ are loaded $\mathrm{K}$ cimes.

\subsection{Granularity}

Assume that parallelism is exploited on submatrix level rather than on block row or block column level. One way to enlarge the degree of parallelism is to change the block size. A reduction of the block size leads to a higher level of parallelism, but not automatically to a higher performance on a parallel machine. A reason for this is that the intensity per block operation - which is of order NB for square blocks-decreases, whereas $M$ and $N$ increase.

Let the cycle time for one floating point operation be $\tau_{\mathrm{f}}$, then for each block $C_{i, j}$ the computation time $T_{\text {c }_{-} \text {block }}$ will be

$$
T_{\mathrm{c}_{-} \text {block }}(k, \mathrm{MB}, \mathrm{NB})=2 \cdot \mathrm{K} \cdot \mathrm{KB} \cdot \mathrm{MB} \cdot \mathrm{NB} \cdot \tau_{\mathrm{f}}=2 k \cdot \mathrm{MB} \cdot \mathrm{NB} \cdot \tau_{\mathrm{f}} .
$$

Define $\tau_{\mathrm{t}}$ as the time to transfer one datum word from main memory to cache or vice versa. To compute one block $C_{i, j}$ requires $\mathrm{K} \cdot \mathrm{KB} \cdot \mathrm{MB}+\mathrm{K} \cdot \mathrm{KB} \cdot \mathrm{NB}+\mathrm{MB} \cdot \mathrm{NB}$ loads and $\mathrm{MB} \cdot \mathrm{NB}$ stores. So, the transfer time $T_{\mathrm{t}_{-} \text {block }}$ will be

$$
T_{\mathrm{t}_{-} \text {block }}(k, \mathrm{MB}, \mathrm{NB})=[k(\mathrm{MB}+\mathrm{NB})+2 \cdot \mathrm{MB} \cdot \mathrm{NB}] \tau_{\mathrm{t}} .
$$


The total time $T_{\text {block }}$ needed for computing one block, including loads and stores, is given by

$$
\begin{aligned}
T_{\text {block }}(k, \mathrm{MB}, \mathrm{NB}) & =T_{\mathrm{c}_{-} \text {block }}+T_{\mathrm{t}_{-} \text {block }} \\
& =2 k \cdot \mathrm{MB} \cdot \mathrm{NB} \cdot \tau_{\mathrm{f}}+[k(\mathrm{MB}+\mathrm{NB})+2 \cdot \mathrm{MB} \cdot \mathrm{NB}] \tau_{\mathrm{t}} .
\end{aligned}
$$

The APP's uniprocessor time needed to carry out the matrix-matrix multiplication $C=A * B$ will be

$$
T_{\text {matrix }}=\mathrm{M} \cdot \mathrm{N} \cdot T_{\text {block }}(k, \mathrm{NB}, \mathrm{MB})=2 k m n \tau_{\mathrm{f}}+k(m \cdot \mathrm{N}+\mathrm{M} \cdot n) \tau_{\mathrm{t}}+2 m n \tau_{\mathrm{t}} .
$$

A change of block size affects (i) the time required to load the matrices $A$ and $B$ and (ii) the maximum degree of parallelism. For the given algorithm, halving the block size causes a doubling of the load time for $\boldsymbol{A}$ and $\boldsymbol{B}$ and a change of $2^{2}$ in the degree of maximum parallelism. From (3.2) we may conclude that the uniprocessor time for a matrix-matrix multiplication is minimal for $\mathrm{M}$ and $\mathrm{N}$ as small as possible. In other words, as the cache content increases, the processing time will decrease. Given the cache size, we can create a matrix-matrix multiplication of maximal performance. On the other hand, (3.2) does not indicate for a fixed $m$ and $n$, and a given number of processors, for which block size the lowest wall clock time can be reached.

\subsection{Bus configuration}

Until now, we have not considered the important restriction on the bus configuration: processors on the same bus cannot communicate with the main memory concurrently. If the computation time is much larger than the transfer time this restriction will have a very limited effect. If not, processors will become idle because the required data are not available. For simplicity, we assume that there are $p$ processors $p_{1}, p_{2}, \ldots, p_{p}$ on one bus and that to each processor exactly one block $C_{i, j}$ is assigned. The process of computation and load/store traffic on a processor is shown in Fig. 5.

The process on processor $p_{1}$ computing $C_{i_{1}, j_{1}}$, can be described as follows:

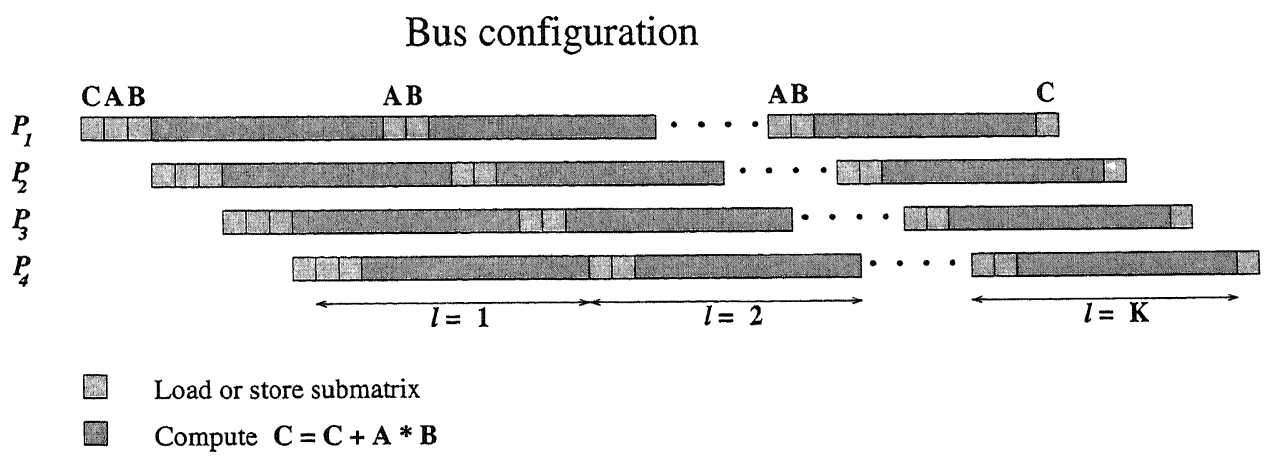

Fig. 5. The load/store and computation process for the matrix-matrix computation $C=C+A * B$ on four processors sharing the same bus. 
- load block matrix $C_{i_{1}, j_{1}}$,

- load block matrices $\boldsymbol{A}_{i_{1}, 1}$ and $\boldsymbol{B}_{1, j_{1}}$,

- compute $\boldsymbol{C}_{i_{1}, j_{1}}=\boldsymbol{C}_{i_{1}, j_{1}}+\boldsymbol{A}_{i_{1}, 1} * \boldsymbol{B}_{1, j_{1}}$.

In the next step, data of $\boldsymbol{C}_{i_{1}, j_{1}}$ can remain in cache, and therefore, only $\boldsymbol{A}_{i_{1}, 2}$ and $\boldsymbol{B}_{2, j_{1}}$ must be loaded. After $\mathrm{K}$ steps the process ends by storing the final $\boldsymbol{C}_{i_{1}, j_{1}}$ data. The computation of submatrix $C_{i_{2}, j_{2}}$ on processor $p_{2}$ requires three blocks to be loaded, too. The best solution is to load $\boldsymbol{C}_{i_{1}, j_{1}}, \boldsymbol{A}_{i_{1}, 1}$ and $\boldsymbol{B}_{1, j_{1}}$ on $p_{1}$ and next, simultaneously, $p_{1}$ starts executing $\boldsymbol{C}_{i_{1}, j_{1}}=\boldsymbol{C}_{i_{1}, j_{1}}+$ $\boldsymbol{A}_{i_{1}, 1} * \boldsymbol{B}_{1, j_{1}}$ and $p_{2}$ is loaded with data of $\boldsymbol{C}_{i_{2}, j_{2}}, \boldsymbol{A}_{i_{2}, 1}$ and $\boldsymbol{B}_{1, j_{2}}$. Processor $p_{2}$ starts updating $\boldsymbol{C}_{i_{2}, j_{2}}$ as soon as all data required are available in cache. The overhead of computing $\boldsymbol{C}_{i_{1}, j_{1}}$ and $C_{i_{2}, j_{2}}$ simultaneously, on two processors sharing the same bus, is equal to the time required for loading $\boldsymbol{A}_{i_{1}, 1}, \boldsymbol{B}_{1, j_{1}}$ and $\boldsymbol{C}_{i_{1}, j_{1}}$ under the restriction that the time needed for loading is less than the computation time for $\boldsymbol{C}_{i_{1}, j_{1}}=\boldsymbol{C}_{i_{1}, j_{1}}+\boldsymbol{A}_{i_{2}, 1} * \boldsymbol{B}_{1, j_{1}}$.

We can extend this process to $p$ processors sharing the same bus. The overhead can be expressed by

$$
(p-1)[\mathrm{KB} \cdot \mathrm{MB}+\mathrm{KB} \cdot \mathrm{NB}+\mathrm{MB} \cdot \mathrm{NB}] \cdot \tau_{\mathrm{t}} .
$$

If this time exceeds

$$
2 \cdot \mathrm{KB} \cdot \mathrm{MB} \cdot \mathrm{NB} \cdot \tau_{\mathrm{f}},
$$

then more than one processor will try to communicate with the main memory at the same time and processors will become idle. Note the significance of the ratio $\tau_{\mathrm{f}}: \tau_{\mathrm{t}}$.

The maximum useful number of processors on a bus can be reflected in the leverage value, which is the ratio of the compute time to data transfer time. A leverage value $l$ indicates that $l+1$ processors can share the same bus, effectively. In other words, the wall clock time cannot be reduced using more than $l+1$ processors. For the matrix-matrix multiplication we obtain

$$
\begin{aligned}
\text { Leverage } & =\frac{\text { Time spent computing in cache }}{\text { Time spent transferring across the bus }} \\
& =\frac{2 \cdot k \cdot \mathrm{NB} \cdot \mathrm{MB} \cdot \tau_{\mathrm{f}}}{[k(\mathrm{MB}+\mathrm{NB})+2 \cdot \mathrm{MB} \cdot \mathrm{NB}] \cdot \tau_{\mathrm{t}}} .
\end{aligned}
$$

We remark that for the example shown in Fig. 5, four processors can share the same bus efficiently. The addition of a fifth processing element on the bus, will not improve efficiency. It is worth noting that, when the computation starts, all values must be available on the on-chip cache; for instance not only the submatrix elements, but also the block size must be known. Since the CRAY APP can be considered as a shared memory machine, all processors have access to the main memory. In case of values being not available on the on-chip cache, a processor will try to load them from main memory. This action will disturb the bus traffic, and processors can become idle resulting in a longer execution time.

\subsection{Numerical experiments with matrix-matrix multiplication}

In this section, the effect of data traffic on the performance is illustrated by means of the matrix-matrix multiplication. The multiplication has a high computation intensity and, therefore, it is possible to increase the performance by using several processors on the same bus. 
It is not our intention to develop the most efficient matrix-matrix multiplication-there exists already a very efficient implementation, i.e. rgmmul, which is appropriate for all kinds of matrix shapes-but in this paper we focus on the performance loss due to bus traffic for some fixed block sizes. A high computation speed is needed in order to demonstrate accurately the influence of data traffic. Therefore, we would like to develop a fast implementation for the matrix-matrix multiplication and to control data traffic explicitly, using APP routines to move data from memory into processor cache and vice versa. It appears that the highest possible performance for a matrix-matrix multiplication in cache can be obtained using the routine _dmmm. Unfortunately, all data traffic is governed internally by the routine (see Section 2.2). We use the routine in order to achieve a high computational efficiency and can only speculate on how the routine manages bus traffic.

To get a clear view on the efficiency we have chosen timing experiments on matrices with sizes depending on the configuration chosen. This enables us to avoid idle time due to a lack of parallelism. For a partitioning of the matrices $A, B$ and $C$ into square blocks of order $b$ we choose

$$
\begin{aligned}
& m=b \times \# \text { processors/bus, } \\
& n=b \times \text { \#buses, } \\
& k=b \times \mathrm{K},
\end{aligned}
$$

for $\mathrm{K}=1, \ldots, 10$ (cf. Fig. 3). This implies that, for a fixed $\mathrm{K}$, the wall clock time for each configuration will be approximately the same. However, the more processing elements involved the higher the Mflop rate will be. Since all cache loads and stores are internal to _dmmm we
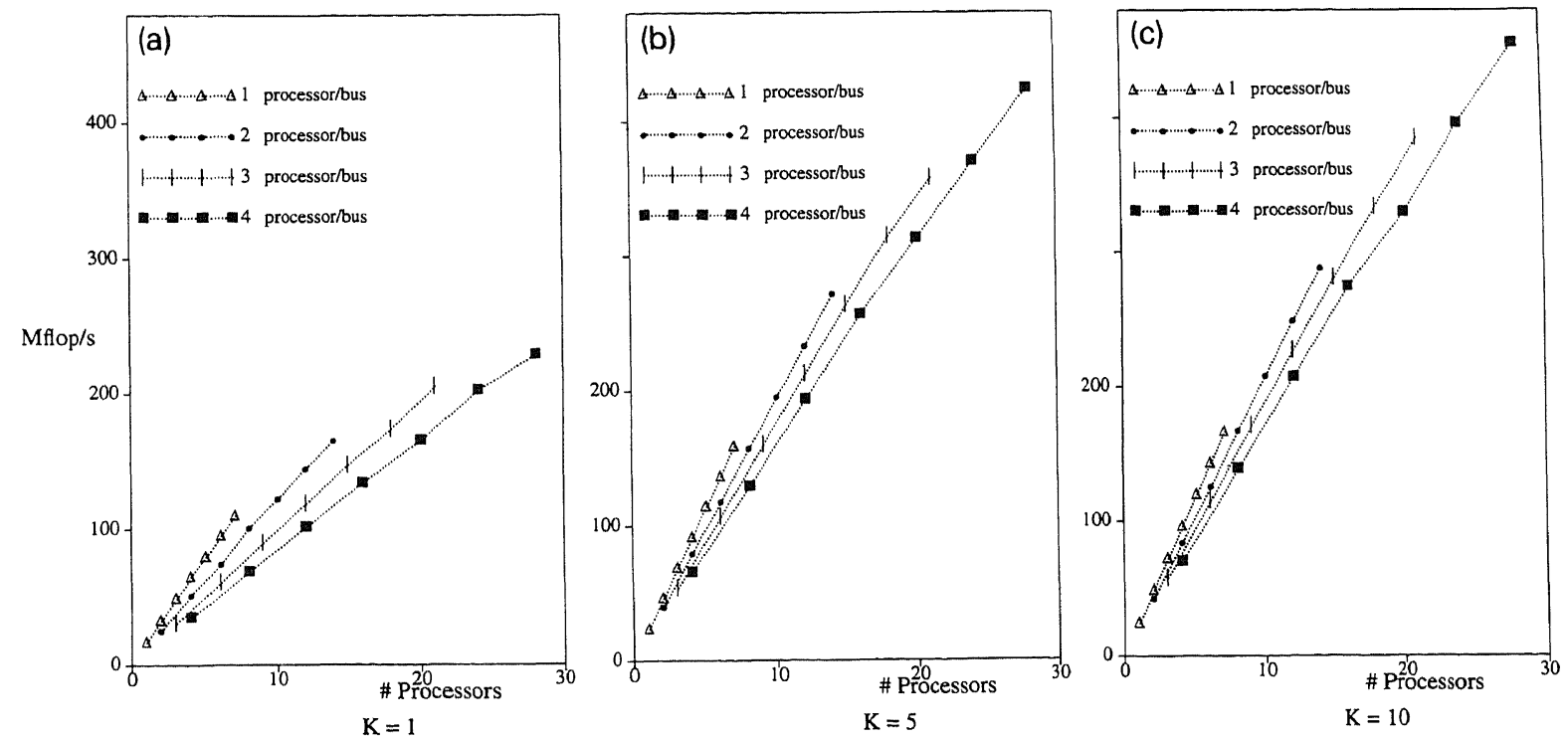

Fig. 6. Floating point rate in Mflop/s achieved for the matrix-matrix product for orders defined by (3.6a)-(3.6c), where $b=18$, the maximum attainable block size. K corresponds with the number of block matrix multiplications per resulting block $C_{i, j}$. 

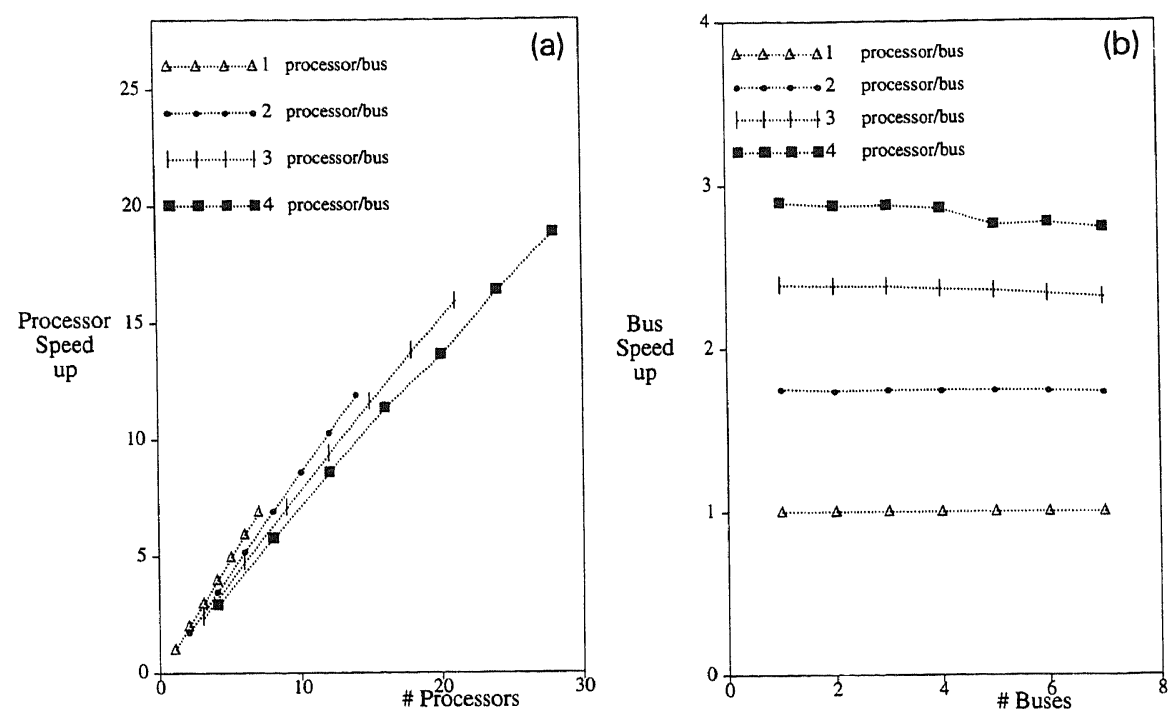

Fig. 7. Processor and bus speed-up for the matrix-matrix product with $\mathrm{K}=10$ and a block size $b=18$.

did not expect any performance improvement by increasing $\mathrm{K}$. Instead of one load and one store for each submatrix $C_{i, j}, \mathrm{~K}$ loads and $\mathrm{K}$ stores are required when preloaded data are lost after a call of _dmmm. Fig. 6 , however, shows that in practice the performance depends on $\mathrm{K}$. Probably, it is recognized during execution, that the elements of $C_{i, j}$ are already in cache.

We have chosen to present the speed-up in two ways:

$$
\begin{aligned}
& S_{\text {processor }}=\frac{\text { Execution time on } 1 \text { processor }}{\text { Execution time on B } \times \mathrm{P} \text { processors }}, \\
& S_{\text {bus }}=\frac{\text { Execution time on B buses with } 1 \text { processor }}{\text { Execution time on } \mathrm{B} \times \mathrm{P} \text { processors }} .
\end{aligned}
$$

For $\mathrm{K}=10$ and block size $b=18$ a processor speed-up close to 18 is reached for the full configuration. The bus speed-up lines in Fig. 7 are nearly horizontal, which implies that the gain of adding more processing elements to a bus does not depend on the number of buses already involved. In other words, in that direction the configuration is scalable. The effect of adding more processing elements to a bus is less obvious. Fig. 8 clearly displays that an increase in cache contents corresponds with an increase of bus speed-up and thus the performance will grow. For this application, it does not pay to use more than three processing elements per bus in case $b=10$. In view of the peak rates for $\tau_{\mathrm{f}}$ and $\tau_{\mathrm{t}}$ as mentioned in Section 2.1, which deliver $\alpha=2$, we could have expected here a leverage value of 5 .

\section{A performance model of the CRAY APP}

From the previous section it is known that when the number of buses increases the speed-up will increase linearly. In contrast, adding more processing elements to a bus will not lead to the 


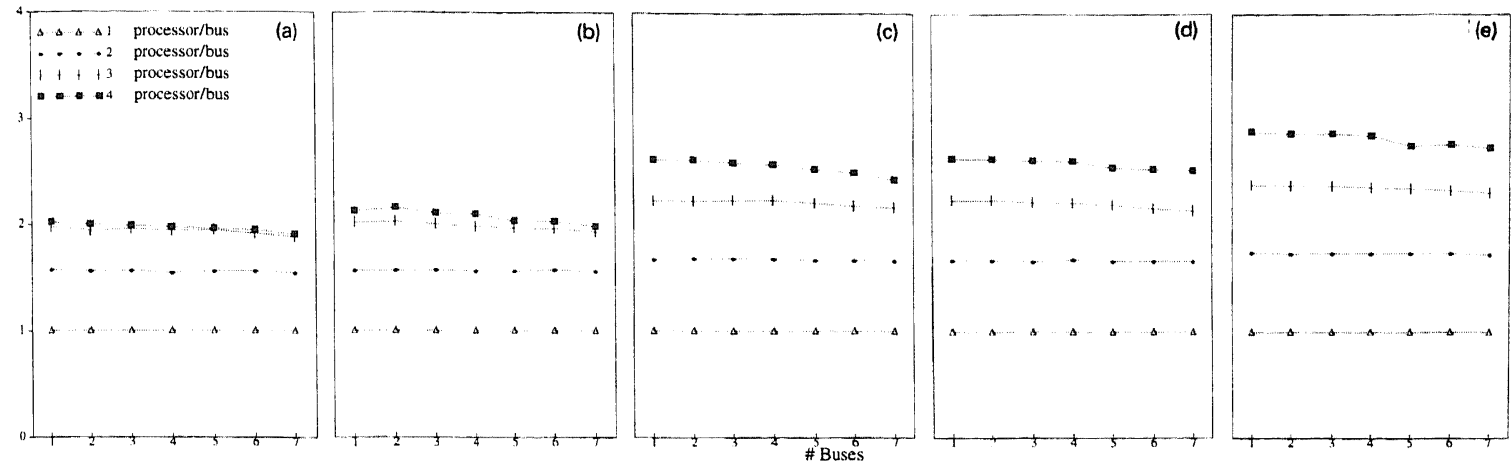

Fig. 8. Influence of bus traffic for the matrix-matrix product $(\mathrm{K}=10)$. From left to right the results of the bus speed-up for block sizes $b=10,12,14,16,18$.

same speed-up. Only a few applications have such a high computation intensity that they can use the APP configuration with four processors per bus efficiently. At least three other characteristics of the APP can be distinguished which determine the ultimate performance result:

- the speed of a processing element,

- the speed of data transfer,

- the cache size of a processing element.

Let $\tau_{\mathrm{t}}$ be the (measured) transfer time for one floating point number and let $\tau_{\mathrm{f}}$ be the (measured) time needed for one floating point operation in SINGLE or DOUBLE PRECISION arithmetic. Assume that $\tau_{\mathrm{t}}$ is a constant independent of the length of the vector to be transferred and that most of the floating point operations can be performed at speed $\tau_{\mathrm{f}}$. In addition, we assume that a sufficient-at least the leverage value (3.5) - number of processing elements is available on a bus. In this section we illustrate how the performance of a bus will change when one of the above mentioned machine quantities is modified. For that purpose, we define $\alpha$ as the ratio of $\tau_{\mathrm{t}}$ to $\tau_{\mathrm{f}}$. The transfer time can then be written as

$$
\tau_{\mathrm{t}}=\alpha \tau_{\mathrm{f}}
$$

As an example we take the matrix-matrix multiplication (cf. Fig. 3) with $m=n=k$, block size $\mathrm{KB}=\mathrm{MB}=\mathrm{NB}=b$ and $\mathrm{K} \times b=k$. The number of floating point operations (flops) per block for this application is given by

$$
\text { \#flops }=2 \mathrm{~K} b^{3}=2 k b^{2} \text {, }
$$

and the number of data transfers by

$$
\text { \#transfers }=2(\mathrm{~K}+1) b^{2} .
$$

The processor performance can be expressed by

$$
\Psi_{\text {processor }}=\frac{\# \text { flops }}{\text { execution time }}=\frac{2 k b^{2}}{2 k b^{2} \tau_{\mathrm{f}}+2(\mathrm{~K}+1) b^{2} \tau_{\mathrm{t}}}=\frac{k}{k+(\mathrm{K}+1) \alpha} \cdot \frac{1}{\tau_{\mathrm{f}}} .
$$
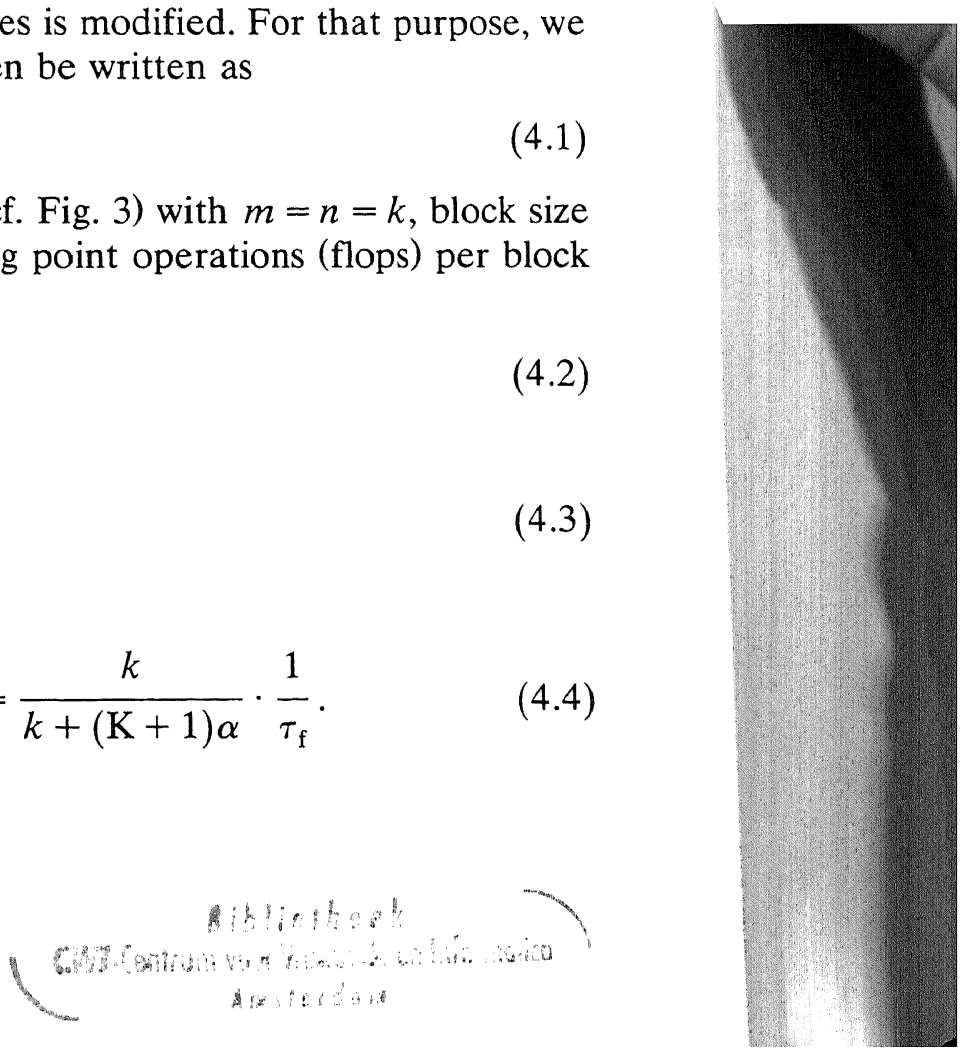
The leverage of the matrix-matrix multiplication (3.5) is denoted by

$$
\text { Leverage }=\frac{2 k b^{2} \tau_{\mathrm{f}}}{2(\mathrm{~K}+1) b^{2} \tau_{\mathrm{t}}}=\frac{k}{(\mathrm{~K}+1) \alpha} .
$$

The maximum performance per bus $\Psi_{\text {bus }}$ is defined by

$$
\Psi_{\text {bus }}=\text { Leverage } \cdot \Psi_{\text {processor }}=\frac{k^{2}}{[k+(\mathrm{K}+1) \alpha](\mathrm{K}+1) \alpha} \cdot \frac{1}{\tau_{\mathrm{f}}} .
$$

If the block size $b$ is chosen such that the data cache is (nearly) fully occupied, the smallest possible value of $\mathrm{K}$ is obtained. This value results in a maximum processor performance and the leverage is maximal, too, and so is the bus performance $\Psi_{\text {bus }}$.

Let us return to the APP characteristics which influence the bus performance. First, we assume that it is possible to increase the speed of a processing element by a factor $\beta, \beta>1$. At the same time we assume that the data transfer time remains constant. In formula,

$$
\left.\begin{array}{l}
\tilde{\tau}_{\mathrm{f}}=\tau_{\mathrm{f}} / \beta \\
\tilde{\tau}_{\mathrm{t}}=\tau_{\mathrm{t}}
\end{array}\right\} \tilde{\alpha}=\frac{\tilde{\tau}_{\mathrm{t}}}{\tilde{\tau}_{\mathrm{f}}}=\alpha \cdot \beta
$$

The ratio of the improved computation speed's bus performance and the actual speed's bus performance becomes

$$
\frac{\tilde{\Psi}_{\text {bus }}}{\Psi_{\text {bus }}}=\frac{k+(\mathrm{K}+1) \alpha}{k+(\mathrm{K}+1) \alpha \beta}<1, \text { for } \beta>1 .
$$

As a very undesirable effect, we get a decrease in bus performance and, consequently, a decrease in APP performance when the speed of the individual processing element increases. Fig. 9 shows an example of an application running efficiently on five processors sharing the same bus. If the computation time is reduced to half the original time, not more than three processors on a bus can be used and the new bus time becomes larger than for the "slow" case.

Secondly, we assume that the transfer rate can be accelerated by a factor $\gamma, \gamma>1$, and the computation speed will be unaltered. Then we get

$$
\tilde{\alpha}=\alpha / \gamma, \quad \tilde{\tau}_{\mathrm{f}}=\tau_{\mathrm{f}}
$$

and the maximum bus performance $\tilde{\Psi}_{\text {bus }}$ becomes

$$
\tilde{\Psi}_{\text {bus }}=\frac{k^{2}}{[\gamma k+(\mathrm{K}+1) \alpha](\mathrm{K}+1) \alpha} \cdot \frac{\gamma^{2}}{\tau_{\mathrm{f}}} .
$$

Compared to the original situation we get an acceleration of

$$
\frac{\tilde{\Psi}_{\text {bus }}}{\Psi_{\text {bus }}}=\gamma \frac{k+(\mathrm{K}+1) \alpha}{k+(\mathrm{K}+1) \alpha / \gamma}>\gamma, \text { for } \gamma>1 .
$$

Without doubt a reduction of the transfer time will improve the performance of the APP. 


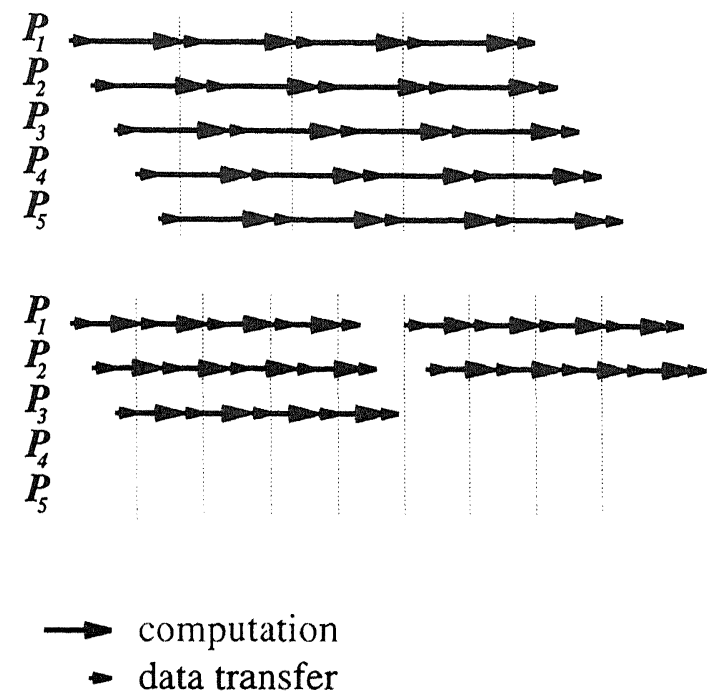

Fig. 9. In the upper part five processors share the same bus efficiently. A reduction in computation time reduces the number of useful processors on the bus. In the new situation only three processors can be used resulting in an increase of the bus time.

Thirdly, we consider the maximum bus performance as a function of the cache contents. If the data cache size is enlarged by $\delta$, then the block size $\tilde{b}$ can be enlarged to

$$
\tilde{b}=\sqrt{\delta} \cdot b
$$

and for a fixed problem, $\tilde{\mathrm{K}}$ will become

$$
\tilde{\mathrm{K}}=\delta^{-1 / 2} \mathrm{~K} \text {. }
$$

The maximum bus performance will increase then by a factor

$$
\frac{\tilde{\Psi}_{\text {hus }}}{\Psi_{\text {bus }}}=\frac{k+(\mathrm{K}+1) \alpha}{k+\left(\delta^{-1 / 2} \mathrm{~K}+1\right) \alpha} \cdot \frac{\mathrm{K}+1}{\delta^{-1 / 2} \mathrm{~K}+1}>1, \quad \text { for } \delta>1,
$$

since both the leverage and the performance per processing element grow. Notice that the gain is less spectacular than obtained for a reduced transfer time.

Summarizing, from the possibilities to improve the bus performance described above the largest gain can be expected by an increase of the transfer speed, whereas a decrease of the cycle time for a floating point operation will even result in a lower bus performance.

\section{The Cholesky factorization}

The block Cholesky matrix factorization has a computational complexity which is comparable to that of the matrix-matrix multiplication. In this section we describe two variants: the block left-looking factorization and the block right-looking factorization. A third variant, the block top-looking, is not considered: for this we expect a similar behavior as for the left-looking 
variant. All variants have exactly the same number of floating point operations. The maj are described in terms of the BLAS $[5,6,10]$ and the organization is such that each p1 will perform BLAS operations on single block matrices only.

The Cholesky factorization of a symmetric, positive-definite matrix $A$ is given by

$$
\boldsymbol{A}=\boldsymbol{L} \cdot \boldsymbol{L}^{\mathrm{T}}
$$

where $L$ is a unique lower triangular matrix. Assume that $A$ can be partitioned into $\mathrm{K}^{2}$ blocks of order $b$ such that $k$, the order of $A$, is equal to $\mathrm{K} \times b$. We first derive th. left-looking variant which is block-column-oriented. Next, the right-looking algorithm is ered.

\subsection{Left-looking algorithm}

This well-known factorization can be derived from the following block-matrix produ

$$
\left[\begin{array}{lll}
\boldsymbol{A}_{11} & & \\
\boldsymbol{A}_{21} & \boldsymbol{A}_{22} & \\
\boldsymbol{A}_{31} & \boldsymbol{A}_{32} & \boldsymbol{A}_{33}
\end{array}\right]=\left[\begin{array}{lll}
\boldsymbol{L}_{11} & & \\
\boldsymbol{L}_{21} & \boldsymbol{L}_{22} & \\
\boldsymbol{L}_{31} & \boldsymbol{L}_{32} & \boldsymbol{L}_{33}
\end{array}\right] \cdot\left[\begin{array}{lll}
\boldsymbol{L}_{11}^{T} & \boldsymbol{L}_{21}^{T} & \boldsymbol{L}_{31}^{T} \\
& \boldsymbol{L}_{22}^{T} & \boldsymbol{L}_{32}^{T} \\
& & \boldsymbol{L}_{33}^{T}
\end{array}\right]
$$

We assume that in the previous $l-1$ steps the block matrices $\boldsymbol{L}_{11}, \boldsymbol{L}_{21}$ and $\boldsymbol{L}_{3}$ consisting of $(l-1) \times b$ columns and $(l-1) \times b$ rows, $b$ rows and $(\mathrm{K}-l) \times b$ rows, resf - have been computed. In the current step $l$, the block matrices $\boldsymbol{L}_{22}$ and $\boldsymbol{L}_{32}$, with a width of $b$ columns, must be updated. Then from the block matrix equality, we obtain

$$
\begin{aligned}
& \boldsymbol{L}_{21} \cdot \boldsymbol{L}_{21}^{\mathrm{T}}+\boldsymbol{L}_{22} \cdot \boldsymbol{L}_{22}^{\mathrm{T}}=\boldsymbol{A}_{22}, \\
& \boldsymbol{L}_{31} \cdot \boldsymbol{L}_{21}^{\mathrm{T}}+\boldsymbol{L}_{32} \cdot \boldsymbol{L}_{22}^{\mathrm{T}}=\boldsymbol{A}_{32} .
\end{aligned}
$$

. ne computation of the diagonal block $L_{22}$ consists of two steps:

$$
A_{22}^{\prime} \leftarrow A_{22}-L_{21} \cdot L_{21}^{\mathrm{T}} \text {, }
$$

or, in Level 3 BLAS terms, a DSYRK operation, followed by

$$
\boldsymbol{L}_{22} \leftarrow \operatorname{Cholesky}\left(\boldsymbol{A}_{22}^{\prime}\right) \text {, }
$$

which can be performed by a Level 2 BLAS algorithm. We refer to this algorithm as The block column matrix $L_{32}$ can be obtained by first computing

$$
A_{32}^{\prime} \leftarrow A_{32}-L_{31} \cdot L_{21}^{\mathrm{T}} \text {, }
$$

a straightforward matrix-matrix multiplication and subtraction, and followed by $\boldsymbol{L}_{32}$

$$
\boldsymbol{L}_{32} \leftarrow \boldsymbol{A}_{32}^{\prime} \cdot \boldsymbol{L}_{22}^{-\mathrm{T}} \text {. }
$$

In (5.7) a triangular system is solved with a multiple right-hand side, which can be perfc the Level 3 BLAS routine DTRSM. In the next step another block column of $L$ is upda after precisely $\mathrm{K}$ steps the factorization is completed. This algorithm is known as a lefi algorithm because the data referred to is mainly on the left-hand side of the curre column. 
square

: block

sonsid-

\subsubsection{Explicit parallelism by operating on single blocks}

The BLAS operations can be parallelized either implicitly or explicitly. In case of implicit parallel processing the BLAS are parallelized such that within a BLAS operation the work is equally divided over several processors. In case of explicit parallel processing, calculations on a single block - both input and output matrices consists of single blocks of dimension $b$-are not parallelized; however, these block operations themselves, scheduled more or less arbitrarily (taking into account the data dependencies) can be performed in parallel.

In [11] parallelism is exploited in a dynamic way. Before the computation starts, the dependencies between single block operations are uniquely described and when a processor has accomplished some single block operation then it "looks" for another operation in a queue of "ready-to-start" processes. After completion of a block operation other processes can become "ready-to-start". This asynchronous approach makes it possible to reduce idle time substantially and, therefore, a highly efficient method is obtained. An important disadvantage of this method, however, is that an extensive administration of the state of the processes and the dependencies is required. SCHEDULE [4], the package that was used for that purpose in [11], is not available on the CRAY APP. Therefore, parallelism is considered at a lower level: a simpler static scheduling of operations is applied, although reduction of idle time is still one of the main issues.

\subsubsection{Parallel implementation}

To start with we discuss how the multiple block operations (5.4), (5.6) and (5.7) can be written as single block operations and how parallelism can be introduced explicitly. The symmetric rank- $k$ update (5.4) can be rewritten as $l-1$ single block DSYRK operations. Since all of the operations write to the same data block $A_{22}^{\prime}$, they cannot be carried out concurrently. Furthermore, the matrix-matrix multiplication (5.6) can be split up into $(\mathrm{K}-l) \times(l-1)$ single block DGEMM multiplications of which the explicit level of parallelism is only $\mathrm{K}-l$. Finally, the multiple block operation DTRSM (5.7) can be partitioned into $\mathrm{K}-l$ single block operations to be performed simultaneously.

Obviously, the load balancing of such a straightforward implementation is far from optimal. A better balancing is achieved by performing the matrix-matrix multiplication DGEMM and the symmetric rank- $k$ update DSYRK simultaneously. These processes are data-independent: DSYRK updates the diagonal blocks whereas DGEMM writes to off-diagonal blocks (cf. Fig. 10). This approach is satisfactory if the execution time of a block multiplication is comparable with the execution time for a single block symmetric rank- $k$ update. If not, then a better solution can be achieved when a "WHILE" construction, illustrated in Fig. 11, is applied, especially when the number of blocks in the $l$ th column is larger than the number of processing elements $p$. A critical section is used to allow processors to update the shared variable JJ. The MCP_BARRIER is called to guarantee that all operations are finished when the final update of the off-diagonal blocks, performed by DTRSM, starts. This "WHILE" construction makes it possible to execute processes asynchronously. As soon as a sequence of DSYRK or DGEMM is terminated, the processor starts with another operation. Moreover, the sequence of DSYRK operations can immediately be followed by a call of DLLT, since its input is independent of the operations on the off-diagonal blocks in column $l$. We remark that the idle time can be reduced even more by performing the DTRSM immediately after the $(l-1)$ th DGEMM contribution 


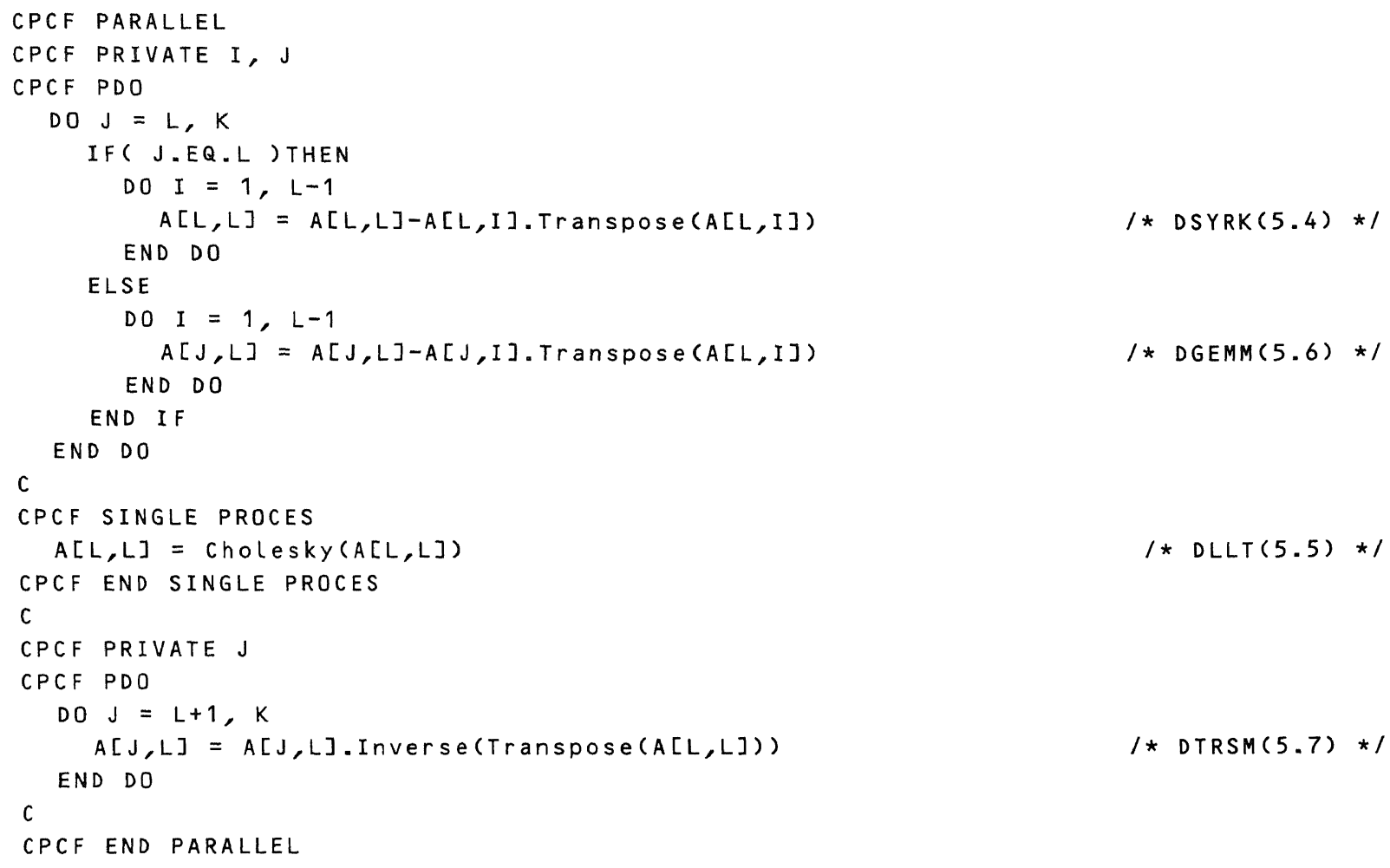

Fig. 10. A pseudo-algorithmic description of the parallel implementation for the $l$ th column of left-looking Cholesky factorization. While one processor updates the diagonal block, other processors perform single block matrix-matrix multiplications.

under the condition that the DLLT on the diagonal block has been completed. However, a comprehensive registration of the status of the processes will be required then.

\subsubsection{Implementation and performance of single block $B L A S$}

Programming the CRAY APP has been reduced to implementing the four basic single block operations: DSYRK, DGEMM, DTRSM and DLLT. As mentioned in Section 2.2, local BLAS implementations as described in [7] are not available, but it is possible to get pipeline rather than scalar performance by means of a very restricted set of Extended Math. Library routines [1].

Both the matrix-matrix product DGEMM and the symmetric rank- $k$ update DSYRK are erformed by a call to _dmmm (see Section 2.2). For the latter this implies that the symmetry if the resulting matrix is not taken into account and twice as many floating point operations are ierformed as actually required. Other attempts to get a better performance for DSYRK failed. Zonsiderable effort has been put in the efficient implementation of DTRSM and DLLT. Table shows the performance in Mflop/s for the single block operations achieved for the optimum lock size $b=18$. We consider the performance given in Table 1 as rather disappointing. 


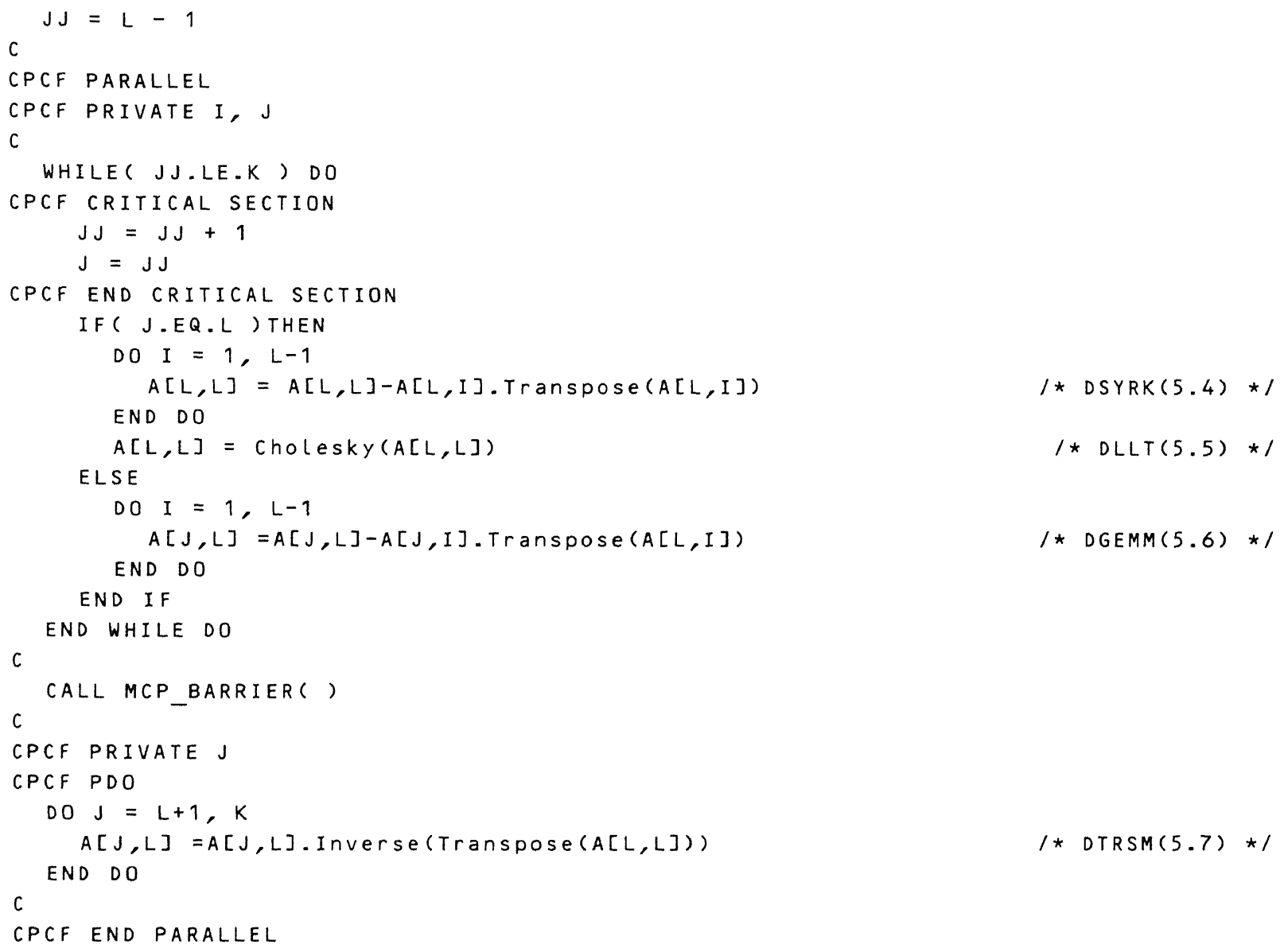

$A[L, L]=$ Cholesky $(A[L, L]) \quad / * D L L T(5.5) * /$

/* DSYRK(5.4) */

/* $\operatorname{DGEMM(5.6)*/~}$

Fig. 11. A pseudo-algorithmic description of the parallel implementation for the $l$ th column of the left-looking Cholesky factorization with the "WHILE" construction. The update of the diagonal block and those of the off-diagonal blocks are carried out concurrently. As soon as either a diagonal or an off-diagonal block update is terminated, the processor may continue with updating another off-diagonal block.

Table 1

Performance results and number of floating point operations of local BLAS operations for the optimum block size $b=18$

\begin{tabular}{lrl}
\hline Block routine & Performance & \multicolumn{1}{c}{ \# flops } \\
\hline DGEMM & $25.6 \mathrm{Mflop} / \mathrm{s}$ & $2 b^{3}$ \\
DSYRK & $13.6 \mathrm{Mflop} / \mathrm{s}$ & $b^{3}$ \\
DTRSM & $6.7 \mathrm{Mflop} / \mathrm{s}$ & $b^{3}$ \\
DLLT & $4.0 \mathrm{Mflop} / \mathrm{s}$ & $\left(2 b^{3}+3 b^{2}+b\right) / 6$ \\
\hline
\end{tabular}



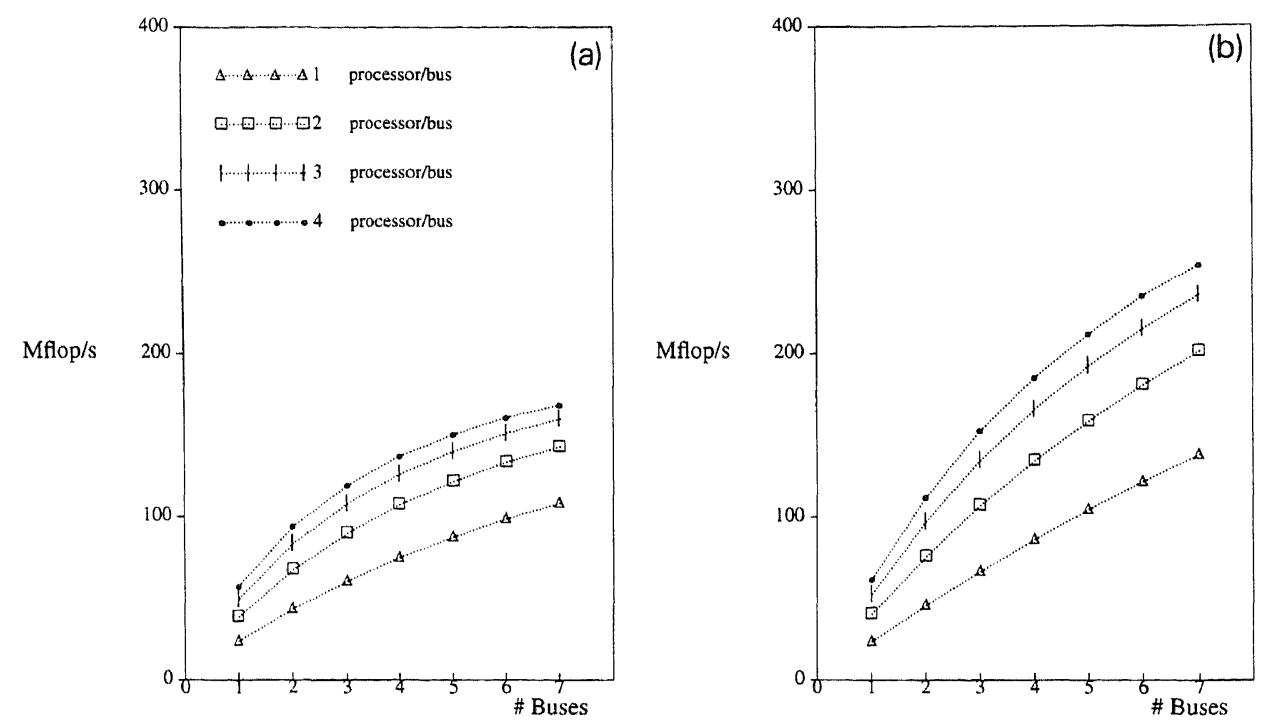

Fig. 12. Performance results of two left-looking block Cholesky factorizations. The left figure corresponds to the implementation presented in Fig. 10, whereas the right figure shows the Mflop rate achieved for the "WHILE" construction of Fig. 11.

\subsubsection{Performance of left-looking implementations}

Fig. 12 shows the performance results obtained for two left-looking variants: the left figure for the implementation presented in Fig. 10, the right figure for the implementation presented in Fig. 11. So, for the left-looking block Cholesky factorization, with its decreasing amount of computational work per block column, asynchronous job scheduling can improve the performance substantially. Moreover, the performance gain obtained by going from 3 to 4 processors per bus is less obvious than from 1 to 2 processors per bus. However, an increase of the total number of processors may not result in a performance increase. For instance, the execution time achieved on 14 processors (viz., 7 buses $\times 2$ processors/bus) is less than the time achieved on 15 processors (viz., 5 buses $\times 3$ processors /bus) for both implementations. In Fig. 13, we present the bus efficiency, defined by the ratio of bus speed-up (3.7) to the number of processors per bus. Although the same steps are performed, we observe that due to a better job scheduling the bus efficiency for the full configuration has been raised from less than $30 \%$ to nearly $40 \%$.

\subsection{Right-looking algorithm}

The block right-looking algorithm has an updating pattern which significantly differs from the left-looking algorithm. The latter does not perform operations before they are actually needed; information from the past - at the left side of the current column - is used to update that column. The right-looking algorithm, however, processes information as soon as it becomes available. If, for instance, the first block column of $\boldsymbol{L}$ has been computed, then all blocks at the 

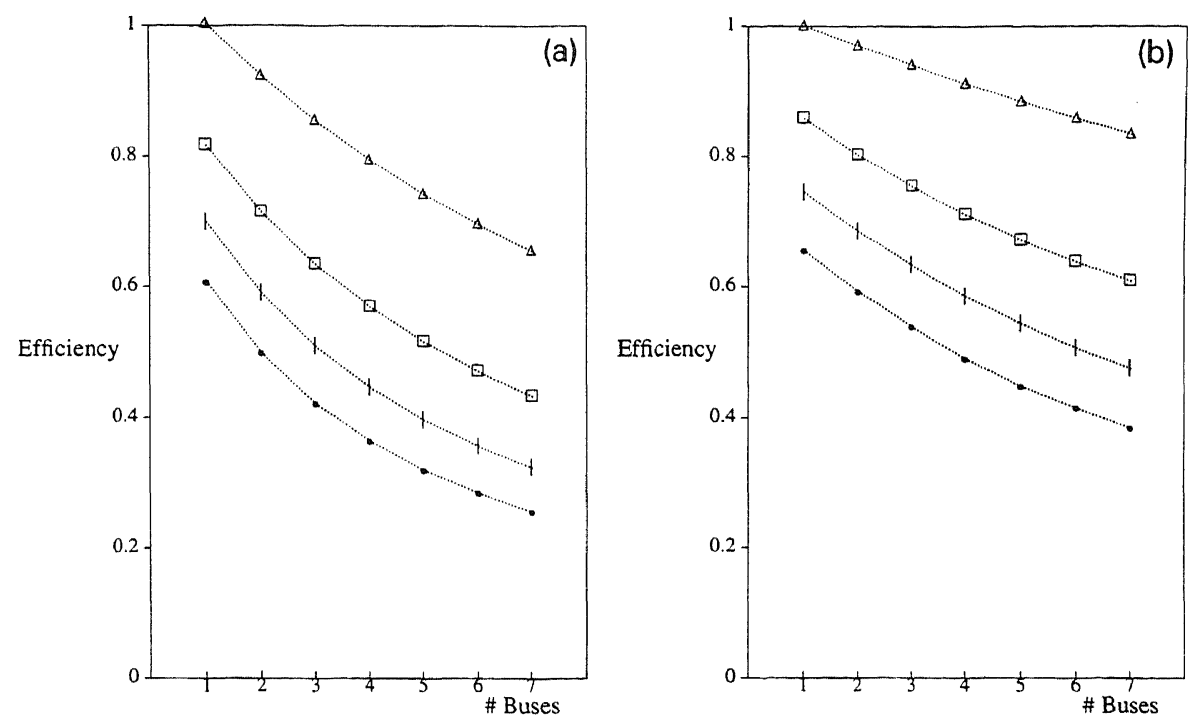

Fig. 13. Bus efficiency for the two left-looking block Cholesky factorizations of Fig. 12.

right-hand side can be updated with contributions from the first block column: the diagonal blocks by a DSYRK operation and the off-diagonal matrices by a matrix-matrix multiplication.

We prefer to describe the right-looking implementation in terms of single block operations directly. Again it is assumed that $\boldsymbol{A}$ (and $\boldsymbol{L}$ ) are of order $k$ and $k=\mathrm{K} \times b$, where $b$ denotes the block size. In the first step the factorization can be performed analogously to the left-looking algorithm:

$$
\begin{aligned}
& \boldsymbol{L}[1,1] \leftarrow \operatorname{Cholesky}(\boldsymbol{A}[1,1]), \\
& \boldsymbol{L}[i, 1] \leftarrow \boldsymbol{A}[i, 1] \cdot \boldsymbol{L}[1,1]^{-\mathrm{T}},
\end{aligned}
$$

for $i=2, \ldots, \mathrm{K}$. For the remaining part of $A$ the diagonal blocks $A[i, i], i=2, \ldots, \mathrm{K}$, are updated with respect to the first block column of $\boldsymbol{L}$ :

$$
\boldsymbol{A}[i, i]=\boldsymbol{A}[i, i]-\boldsymbol{L}[i, 1] \cdot \boldsymbol{L}[i, 1]^{\mathrm{T}},
$$

and the off-diagonal elements by a matrix-matrix multiply and add operation

$$
\boldsymbol{A}[i, j]=\boldsymbol{A}[i, j]-\boldsymbol{L}[i, 1] \cdot \boldsymbol{L}[j, 1]^{\mathrm{T}},
$$

for $i=3, \ldots, \mathrm{K}, j=2, \ldots, i-1$. As soon as the first step is completed, the same operations can be applied on the recently updated submatrix of $\boldsymbol{A}$. The operations of the first two steps are illustrated by Fig. 14. In Fig. 16 the implementation of the computation of the $l$ th block column and the update of its remaining right-hand part is given. Note that some idle time can be saved by combining DTRSM, DSYRK and the Cholesky factorization of the current block as is shown in Fig. 15. Actually, the reduction of idle time will be larger than shown, because relatively expensive operations are combined. 

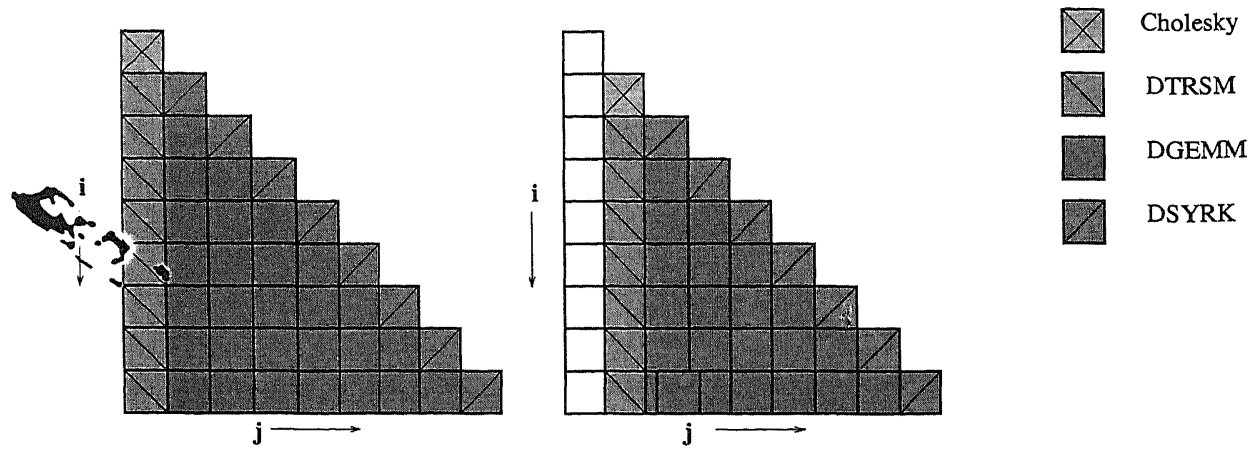

Fig. 14. Update scheme for the first two steps of right-looking block Cholesky factorization.

\subsubsection{Performance of right-looking implementation}

The main difference in performance for the left- and right-looking implementations comes from the level of parallelism. For the left-looking algorithm the number of independent processes for the $l$ th block column is equal to the number of blocks in that column, viz., $\mathrm{K}-l$, in case diagonal and off-diagonal blocks are updated concurrently. For the right looking algorithm this number is much higher, viz.,

$$
\frac{1}{2}(\mathrm{~K}-l) \cdot(\mathrm{K}-l+1),
$$

ince all DSYRK and DGEMM updates are data-independent.

Fig. 17 displays performance results of the block right-looking variant. The results for a small umber of processors are comparable to the left-looking implementations, but for a larger
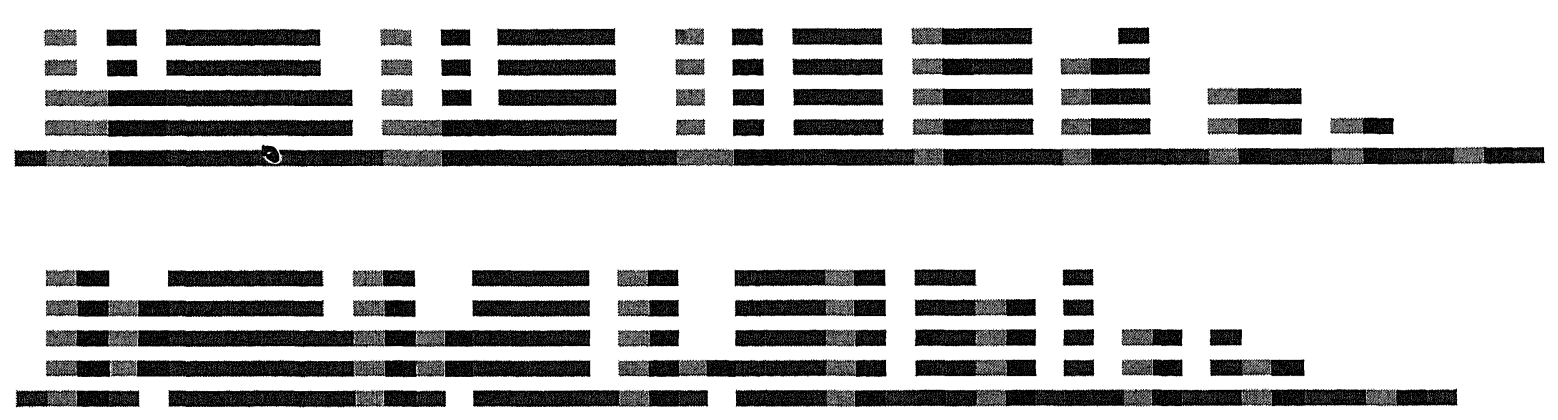

\section{- Cholesky \\ - DSYRK \\ - DTRSM \\ - DGEMM}

Fig. 15. Right-looking block Cholesky factorizations on five processors. The upper pattern illustrates the parallel execution of Fig. 14's implementation. A combination of DSYRK and DTRSM and the Cholesky operations can reduce idle time as is illustrated by the lower pattern. 
s comes

pendent , $\mathrm{K}-\mathrm{l}$, looking

a small larger

parallel ons can

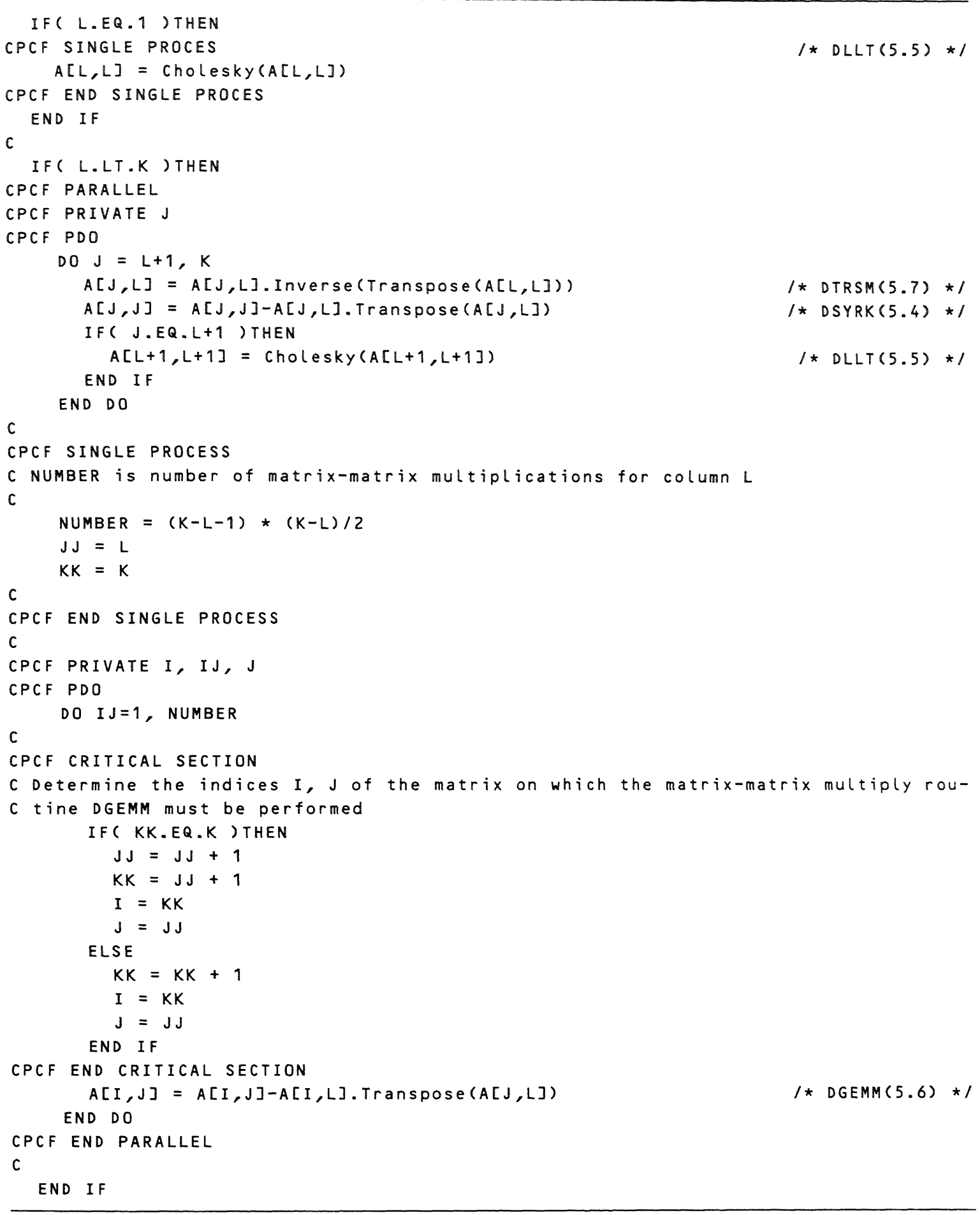

Fig. 16. A pseudo-algorithmic description of the parallel implementation of the right-looking block Cholesky factorization. 

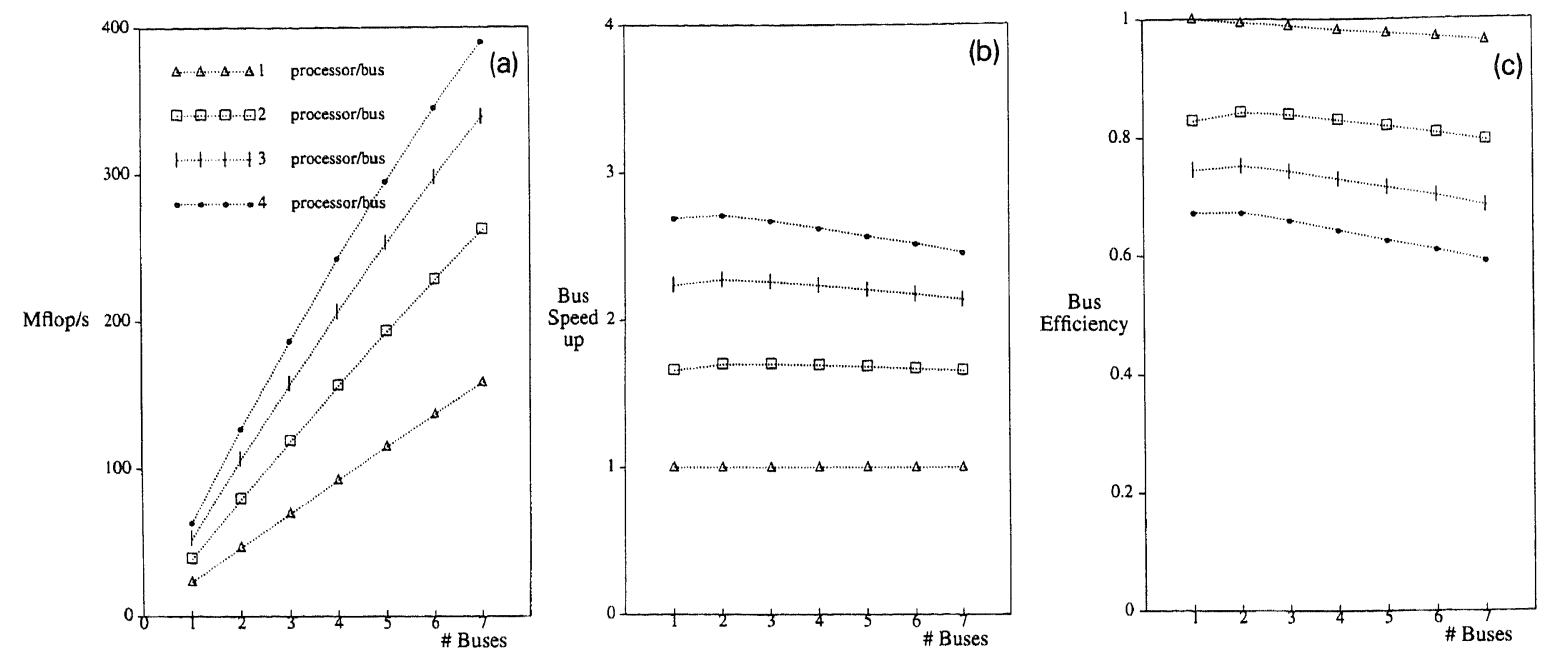

Fig. 17. Results of the right-looking block Cholesky factorization. From left to right: the Mflop/s, the bus speed-up and the bus efficiency for $b=18$, respectively.

number of processors we see that the right-looking implementation performs much better due to the higher level of parallelism. The speed-up pattern approximates the one achieved for the matrix-matrix multiplication (cf. Fig. 8). We also refer to [2] and [11] for similar experiments.

It is worth noting, however, that for the left-looking algorithm the data reuse is better: only data from the current block is updated and can be kept in cache and as we have seen in Section 3.4: this will probably happen. For the right-looking algorithm no data can be reused and, consequently, more bus traffic takes place. Apparently, this disadvantage does not play an mportant role.

Finally, we discuss performance results for different block sizes. In Fig. 18 all lower lines correspond to a block size of 10 , the upper lines to the optimum block size of 18 , the middle
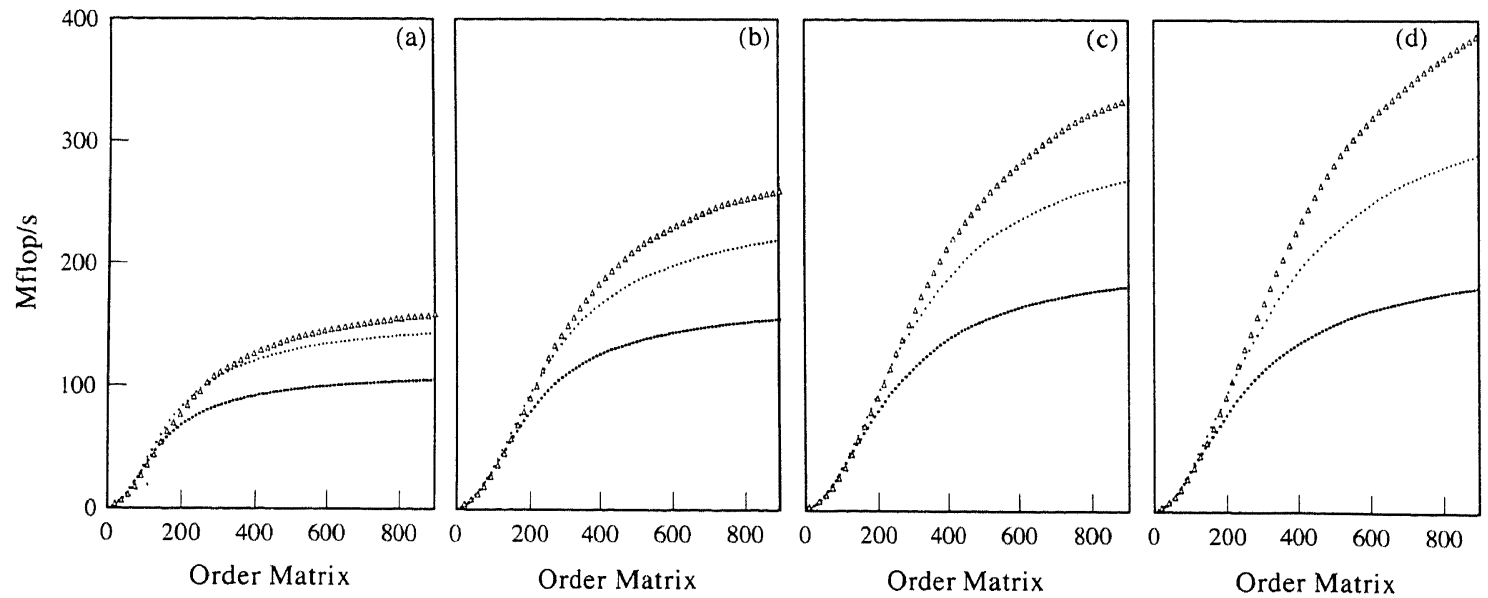

Fig. 18. Performance of the right-looking Cholesky factorization for block sizes: $b=10,16,18$. From left to right the Mflop/s obtained for runs on $7 \times 1,7 \times 2,7 \times 3,7 \times 4$ processors. 
line in each picture corresponds to a block size of 16 . Horizontally, the order of the matrix is displayed. For a fixed matrix order a decrease of the block size delivers a higher level of parallelism, which is not translated into a higher efficiency. From Fig. 18 it is clear that for a block size of 10 the bus traffic negatively influences the performances, whereas a larger block size with a corresponding higher matrix-matrix multiplication's leverage value takes care of a high speed on the full bus configuration.

\section{Conclusions}

We have shown the influence of bus traffic on the APP performance by means of simple block matrix-matrix products. A bus speed-up of nearly three was achieved for matrix-matrix multiplication and optimally filled cache. We do not expect that better results can be obtained with the addition of more processing elements on the same bus.

For the Cholesky decomposition a reasonable efficiency was achieved, not only because of the high matrix-matrix multiplication performance but also because of the choice of algorithm. The right-looking implementation with its higher level of parallelism performs significantly better than the left-looking implementations. For both algorithms the number of synchronization points is significant. By means of taking different jobs together with the so-called "WHILE" construction considerable execution time reduction can be achieved. The best results for the APP configuration were obtained for partitionings with cache contents as large as possible. A partitioning into smaller blocks with a corresponding higher level of parallelism does not lead to a better performance (as is shown by Fig. 18) because of increased data traffic.

\section{Acknowledgements}

The author wishes to thank Alan Stewart and Herman J.J. te Riele for their constructive comments.

\section{References}

[1] Cray Research Superservers, Inc., CRAY APP Programmer's Reference (1992) Extended Math. Routines.

[2] K. Dackland, E. Elmroth, B. Kågström and C. Van Loan, Parallel block matrix factorizations on the shared-memory multiprocessor IBM $3090 \mathrm{vf} / 600 \mathrm{j}$, Internat. J. of Supercomputer Appl. 6 (1992) 69-97.

[3] J.J. Dongarra, Performance of various computers using standard linear equations software in a Fortran environment, Technical Report CS-89-85, Oak Ridge National Laboratory (1992).

[4] F.B. Hanson and D.C. Sorensen, The SCHEDULE parallel programming package with recycling job queues and iterated dependency graphs, Technical Report ANL-MCS-P22-0189, Argonne National Laboratory (1989).

[5] J.J. Dongarra, J. Du Croz, I. Duff and S. Hammerling, A set of level 3 Basic Linear Algebra Subprograms, ACM Trans. Math. Software 16 (1) (1990) 1-17.

[6] J.J. Dongarra, J. Du Croz, S. Hammerling and R.J. Hanson, An extended set of Fortran Basic Linear Algebra Subprograms, ACM Trans. Math. Software 14 (1) (1988) 1-32.

[7] S.L. Johnsson and L.F. Ortiz, Local Basic Linear Algebra Subroutines LBLAS for distributed memory architectures and languages with array syntax, Internat. J. Supercomputer Appl. 6 (1992) 322-350. 
[8] C.-H. Lai and H.J.J. te Riele, Some experiences of solving 1-D semiconductor device equations on a matrix coprocessor by a domain decomposition method, Technical Report NM-R9304, CWI, Amsterdam (1993).

[9] C.-H. Lai, H.J.J. te Riele and A. Ualit, Parallel experiments with simple linear algebra operations on a Cray S-MP System 500 matrix coprocessor, Technical Report NM-N9301, CWI, Amsterdam (1993).

[10] C.L. Lawson, R.J. Hanson, D. Kincaid and F.T. Krogh, Basic Linear Algebra Subprograms for FORTRAN usage, ACM Trans. Math. Software 5 (1979) 308-323.

[11] M. Louter-Nool, Block-Cholesky for parallel processing, Appl. Numer. Math. 10 (1992) 37-57.

[12] A. Stewart, M. Louter-Nool, H.J.J. te Riele and D.T. Winter, An investigation of data reuse on the Cray S-MP System 500, Supercomputer XI (1) 59 (1994/95).

[13] A.J. van der Steen. Overview of recent supercomputers, Technical Report, 5th rev. ed., Stichting Nationale Computer Faciliteiten, The Hague (1995). 